\title{
Giant Sialolith with 20 Years of Evolution in the Submandibular Duct
}

\author{
Victor Tieghi Neto* ${ }^{(\mathbb{D}}$, Rosana Mara Adami Tucunduva $\left(\mathbb{D}\right.$, Andrea Guedes Barreto Gonçales $\left.{ }^{(}\right)$, \\ Eduardo Stedile Fiamoncini, Caio Peres Bellato, Izabel Regina Fischer Rubira-Bullen (1), \\ Eduardo Sanches Gonçales
}

Department of Surgery, Pathology, Radiology and Stomatology, Bauru School of Dentistry, University of Sao Paulo, Bauru, Brazil

Email: ^tieghineto@gmail.com

How to cite this paper: Neto, V.T., Tucunduva, R.M.A., Gonçales, A.G.B., Fiamoncini, E.S., Bellato, C.P., Rubira-Bullen, I.R.F. and Gonçales, E.S. (2020) Giant Sialolith with 20 Years of Evolution in the Submandibular Duct. Open Journal of Stomatology, 10, 115120.

https://doi.org/10.4236/ojst.2020.106013

Received: April 24, 2020

Accepted: June 9, 2020

Published: June 12, 2020

Copyright $\odot 2020$ by author(s) and Scientific Research Publishing Inc. This work is licensed under the Creative Commons Attribution International License (CC BY 4.0).

http://creativecommons.org/licenses/by/4.0/

\begin{abstract}
Sialolithiasis is a common disease that affects the major salivary glands, which is characterized by obstruction of the gland itself or its excretory duct due to the formation of a sialolith, resulting in decreased salivary flow. It mainly affects the submandibular glands $(80 \%-90 \%)$, probably because it has a long duct that surrounds the mylohyoid muscle and emerges against gravity through the Wharton's duct. The size of the salivary calculus can vary from below 1 millimeter $(\mathrm{mm})$ to a few centimeters in diameter, where most stay below $10 \mathrm{~mm}$. Only 7\% are greater than $15 \mathrm{~mm}$ and these are classified as giant calculi. This report presents a case of asymptomatic giant sialolith (approximately $60 \mathrm{~mm}$ in diameter) located in the duct of the right submandibular gland, with 20 years of evolution. The treatment of choice was the surgical removal of the sialolith (calculus) through the salivary duct with maintenance of the gland. Although the individual did not present complaints related to the presence of the giant calculi, its removal had a great impact on their quality of life, since a significant respiratory improvement was reported by the individual. The major difference reported was better sleep quality.
\end{abstract}

\section{Keywords}

Salivary Gland Calculi, Salivary Glands, Sialolithiasis

\section{Introduction}

Sialolithiasis is a common disease of the major salivary glands. It is characterized by mechanical obstruction of the gland or its excretory duct due to the deposition of minerals around an organic matrix giving rise to a salivary calculi (sialolith) resulting in a decrease in salivary flow. It mainly affects the submandibular 
gland ( $80 \%$ to $90 \%$ ) followed by the parotid gland (5\% to $20 \%$ ), sublingual gland (1\% to $2 \%)$ and rarely affects the minor glands [1]-[7].

Sialolithiasis can present 3 different manifestations: firstly with total absence of symptomatology, where a sialolith is accidentally found in routine radiographic examinations; also, it presents as intermittent edema and pain preceding meals around the area involving the affected gland. In addition to these, it can manifest through a process of acute suppuration, resulting from secondary bacterial infection accompanied by persistent edema and pain. In some cases, hyperemia and diffuse edema in the floor of the mouth around the submandibular duct may be observed. Moreover, there are reports of dysphagia and dysarthria in cases of giant sialoliths, but these are extremely rare [2] [7].

Most of the calculi have sizes smaller than $10 \mathrm{~mm}$ and only $7.6 \%$ reach sizes greater than $15 \mathrm{~mm}$, being classified as giant calculi, and considered rare with few cases described in the literature. They may also present as single $(70 \%$ $80 \%)$ or multiple fragments [7] [8] [9].

The purpose of the present article was to report a case of rare giant salivary sialolith, located in the submandibular duct, with 20 years of asymptomatic evolution.

\section{Case Report}

A 48 years old white male patient reported swelling in the floor of the mouth, with approximately 20 years of evolution, asymptomatic since its onset. Presenting with diabetes type 2 and taking metformin $850 \mathrm{mg}$ per day. Also, a history of automotive accident 28 years ago, with mandibular symphyseal fracture and bilateral condyle fracture, which were then treated with with intermaxillary fixation.

Upon physical examination, an elevation of the tongue with swelling in the right side of the floor of the mouth was observed, extending from the region of the submandibular duct anteriorly towards the most posterior portion of the floor of the mouth, mobile, normal in color, with a smooth surface and of a hard consistency (Figure 1). During milking maneuver, no saliva drainage was observed through the submandibular duct on the right side.

Panoramic radiograph examination revealed a radiopaque mass with an elongated cylindrical form in the region of Wharton duct of the right submandibular gland, extending from the region of the mental symphysis back to the angle of the mandible (Figure 2).

Considering the clinical history and radiographic findings, the diagnosis of sialolithiasis in the submandibular duct was established, and the proposed treatment was surgical removal of the calculi approached by means of a local incision under general anesthesia, due to its size and posterior extension.

Due to the long period of evolution of the sialolith, associated with the potential chronic complications of diabetes, patients apathy and indifference to all of his conditions, there could be a risk of infection and important changes in the surrounding tissue, both in the structures of the duct and in the salivary gland 
itself. If the gland required removal, such an assessment would be safer under general anesthesia.

Using an electrocautery, a linear incision was made in the mucosa of the floor of the mouth, in the most anterior aspect of the duct, preserving the sublingual caruncle, allowing access to the sialolith (Figure 3 ). The calculus was completely removed in 4 fragments due to its size, measuring approximately $60 \times 15 \mathrm{~mm}$ (Figure 4) leaving the incision site unsutured, which allows healing by secondary intention avoiding duct obliteration. Considering it is an area at risk of developing infectious processes with the formation of abscesses in the floor of the mouth, postoperative antibiotics (Amoxicillin $500 \mathrm{mg}$ ), analgesics (Dipyrone 1 g) and NSAIDS (Nimesulid $100 \mathrm{mg}$ ) were prescribed. After 7 days, the wound showed no signs of infection or bleeding. The patient did not report painful symptoms or discomfort in the initial postoperative period. After 4 months (Figure 5), consistent salivary flow was observed draining through the new patent drainage duct that was created surgically, preserving the physiological function of the affected gland. With the satisfactory evolution of the case, the new outlet of the duct formed after healing had become functional, replacing the sublingual caruncle.

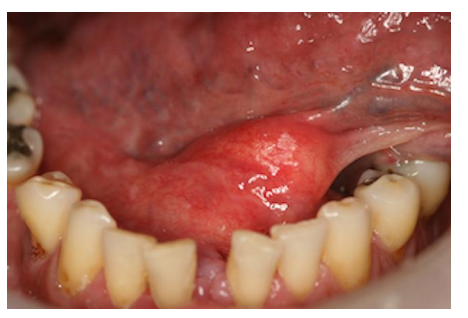

Figure 1. Physical examination a swelling in the floor of the mouth on the right side.

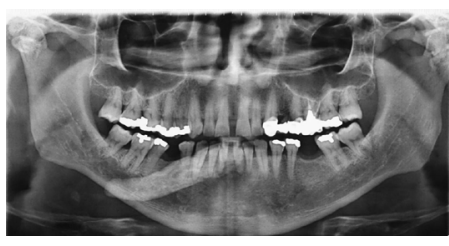

Figure 2. Panoramic radiographic. Radiopaque mass in the region corresponding to the duct of the right submandibular gland, extending from the region of mental symphysis to near the angle of the mandible.

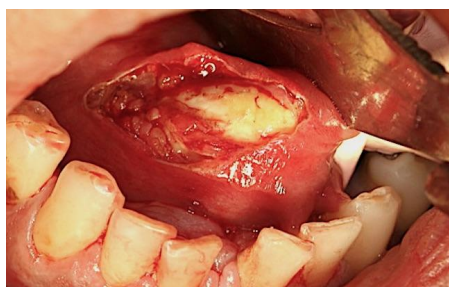

Figure 3. Surgical image of removal of calculi. Access to the calculi through an electrocautery incision. 


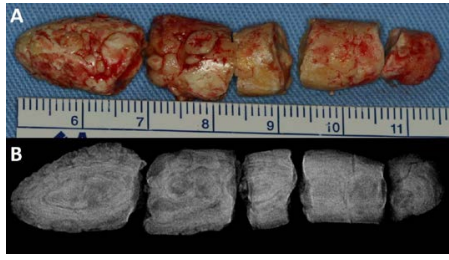

Figure 4. Clinical (A) and radiographic (B) image of the fragments of the calculus. It is possible to observe images that show the calcification layers that form the lesion.

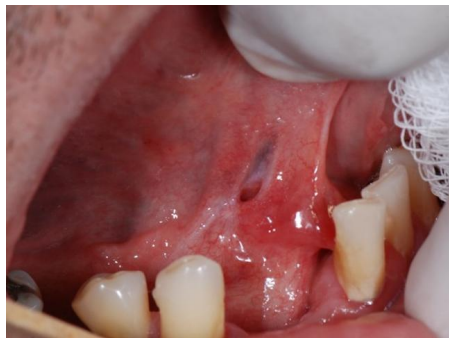

Figure 5. Postoperative period of 4 months. It is possible to notice a new formation of excretory salivary duct.

\section{Discussion}

Sialolithiasis is a relatively common disease of the major salivary glands [1] [2] [7]. Its etiology is still unknown, although there are some theories in the literature that try to explain it. It is believed that due to a change in the composition of saliva by deposition of calcium around an organic matrix, which consists, among other components, of foreign bodies, bacteria and desquamated epithelial cells, an increase of saliva viscosity occurs, which can result in the obstruction of the terminal ducts of the salivary gland [9] [10].

The high rate of occurrence in the submandibular gland can be referred to its anatomical characteristics and long tortuous excretory duct ascending around the mylohyoid muscle (Wharton duct), as well as, a higher concentration of calcium and phosphate salts with relative viscosity of the mucous secretion of this gland [2] [3] [7].

A swelling in the region of the affected gland is the most frequent symptom of sialolithiasis and the main complaint is usually related to pain and edema, during meal periods or when salivary secretion is stimulated. The intensity of the symptoms varies according to the degree of obstruction and the amount of pressure produced inside the gland [2] [10].

It is common for long-term obstructions to lead to infections with subsequent glandular atrophy and alterations in the salivary secretion function and, finally, gland fibrosis [10]. In the present report, the individual has already been informed and educated about his condition, but he never sought treatment. We believe that the slow growth of the sialolith added to the high threshold of tolerance of this patient, caused this negligent behaviour and lack of treatment.

Calcified masses over $10 \mathrm{~mm}$ are considered rare, there are only 14 well documented cases reported in the literature since 1942 [1] [7] [8]. In the present 
case, it was completely removed and in various fragments due to its size of approximately $60 \times 15 \mathrm{~mm}$. If the sialolith-containing duct undergoes dilatation, allowing almost normal salivary secretion, the condition may be asymptomatic over a long period of time and a giant calculus may form [9].

Some diseases, such as tumors, Sjögren's Syndrome, diabetes and alcoholism, can cause persistent decrease of the salivary secretion and are considered in the differential diagnosis of sialolithiasis [8]. Due to the distant time of onset, the effect of the systemic condition and the history of trauma on the formation and development of sialolithiasis remains unknown.

Sialoliths can be found incidentally in panoramic radiographs when there is no symptomatology. The occlusal radiograph is the most indicated for the diagnosis of the calculi located in the anterior $2 / 3$ of the submandibular duct. CT is necessary when the calculi are located in the posterior portion of the duct of the gland or in its interior. It is also the ideal exam for calculi diagnosis in the parotid gland. Sialography is indicated in the case of signs of sialolithiasis, radiolucent calculi or submandibular and deep parotid calculi. Ultrasonography has limited diagnostic value because of its low sensitivity to diagnose small size calculi [2].

The goal of treatment for sialoliths is to restore normal salivary secretion and the treatment may be conservative or, in most cases, surgical, varying according to the affected gland, location and size of the calculi. Like any surgical treatment, it is also possible to develop some complications [3]. However, no intercurrences were observed during the 4-month follow-up of the present report.

Because it did not present painful and infectious symptomatology during its evolution and because it did not present sialolith components inside the gland, it was decided not to remove the right submandibular gland.

Despite not having reported symptoms during the years of evolution of calculi, the individual reported an evident improvement in the quality of life achieved immediately after the procedure, especially related to respiratory function during sleep, eliminating snoring and, consequently, the nocturnal awakenings. He also reported a clear improvement in regard to comfort during meals after removal of calculus. These reports show that the silent complications caused by the presence of this giant calculi could include dysphagia, masticatory difficulties and even apnea or hypopnea in the individual, probably due to the posterior projection of the tongue caused by increased volume due to the presence of the sialolith.

Only one study reported the occurrence of dysphagia and dysarthria related to giant sialolith [7]. Some pathological conditions characterized by a slow evolution may end up altering the perception of some of the symptoms by the individual who ends up adapting to these conditions. It is evident that the role of the assisting health professional is to identify these situations and clarify their potential impact on the general health of the patient, even if the individual does not realize it. 


\section{Conclusion}

The present article showed that when diagnosing large calculi, especially those located posteriorly in the floor of the mouth, the professional should consider they might be associated with serious impairments such as dysphagia and even breathing-related disorders during sleep that are unknown to the patient. Such changes can have more serious repercussions on the patient's general health in the long term than what is originally expected from a sialolith gland obstruction; thus a careful assessment and patient education are key and necessary when opting for the right treatment.

\section{Conflicts of Interest}

The authors declare no conflicts of interest regarding the publication of this paper.

\section{References}

[1] Armstrong, M.A. and Turturro, M.A. (2013) Salivary Gland Emergencies. Emergency Medicine Clinics of North America, 31, 481-499. https://doi.org/10.1016/j.emc.2013.01.004

[2] Mandel, L. (2014) Salivary Gland Disorders. Medical Clinics of North America, 98, 1407-1449. https://doi.org/10.1016/j.mcna.2014.08.008

[3] Lustmann, J., Regev, E. and Melamed, Y. (1990) Sialolithiasis. A Survey on 245 Patients and a Review of the Literature. International Journal of Oral and Maxillofacial Surgery, 19, 135-138. https://doi.org/10.1016/S0901-5027(05)80127-4

[4] Yu, C., Yang, C. and Zheng, L. (2013) Sialendoscopic Findings in Patients with Obstructive Sialadenitis: Long Term Experience. British Journal of Oral and Maxillofacial Surgery, 51, 337-341. https://doi.org/10.1016/j.bjoms.2012.07.013

[5] Dalal, S., Jain, S., Agarwal, S. and Vyas, N. (2013) Surgical Management of an Unusually Large Sialolith of the Wharton's Duct: A Case Report. The Saudi Journal for Dental Research, 4, 33-35. https://doi.org/10.1016/j.ksujds.2012.11.006

[6] Sutay, S., Erdag, T.K., Ikiz, A.O. and Guneri, E.A. (2003) Large Submandibular Gland Calculus with Perforation of the Floor of the Mouth. Otolaryngology-Head and Neck Surgery, 128, 587-588. https://doi.org/10.1016/S0194-5998(02)23280-5

[7] Güngörmüs, M., Yavuz, M.S. and Yolcu, U. (2010) Giant Sublingual Sialolith Leading to Dysphagia. The Journal of Emergency Medicine, 39, e129-e130. https://doi.org/10.1016/j.jemermed.2007.12.026

[8] Zengel, P., Schrotzlmair, F., Reichel, C., Paprottka, P. and Clevert, D.A. (2013) Sonography: The Leading Diagnostic Tool for Diseases of the Salivary Glands. Seminars in Ultrasound, CT and MRI, 34, 196-203.

https://doi.org/10.1053/j.sult.2012.11.012

[9] Bodner, L. and Beer-Sheva (2002) Giant Salivary Gland Calculi: Diagnostic Imaging and Surgical Management. Oral Surgery, Oral Medicine, Oral Pathology, Oral Radiology, and Endodontology, 94, 320-323. https://doi.org/10.1067/moe.2002.123863

[10] Gadve, V., Mohite, A., Bang, K. and Shenoi, S.R. (2016) Unusual Giant Sialolith of Wharton's Duct. Indian Journal of Dentistry, 7, 162-164. https://doi.org/10.4103/0975-962X.174594 